\title{
Einflüsse elektronischer Spiele auf die Entwicklung von mobilen Endgeräten und deren Wert für die Nutzer
}

\author{
Johannes Koslowsky, Danny Janz
}

Zusammenfassung

Diese Arbeit untersucht die Entwicklung von Spielen auf mobile Endgeräten und deren Einfluss auf die zugrunde liegenden Technologien. Es wird auf historische, aktuelle sowie zu erwartende Entwicklungen eingegangen. Außerdem wird gezeigt, was uns erwarten wird und wie dies die Nutzer und die Gesellschaft beeinflussen könnte. Es wird auch die im Rahmen dieser Arbeit durchgeführte Befragung ausgewertet und erläutert. Diese geht der Frage nach, was sich Nutzer in Zukunft auf mobilen Endgeräten wünschen und wie sie mobile Spiele bereits heute nutzen.

\section{Abstract}

The influence of electronic games on mobile phone development and their value for the user This research presents the development of mobile games and their influence to the development of underlying technologies. It will take a look at the beginning of the mobile gaming era and the evolution to the current situation. Furthermore it will present possible future developments. Additionally it tries to give a forecast to what will possibly await us, and the attached value for the user as well as the cultural and ecological aspect. It also allocates the results of a public poll, representing the current user acceptance and what developments the mobile phone and mobile game users would like to see and accept in the future. Especially the acceptance of 3d-Games on mobile platforms is studied.

\section{Einleitung}

Im Rahmen der Lehrveranstaltung Telekommunikation und Gesellschaft des Masterstudiengangs Telematik wurde in dieser Projektarbeit untersucht:

- welche Einflüsse elektronische Spiele auf die Entwicklung von mobilen Endgeräten haben und

- wie sich elektronische Spiele auf mobilen Endgeräten (mobile Games) gesellschaftlich auswirken.

Um dies zu untersuchen, wurde im Zuge dieser Projektarbeit eine quantitative Untersuchung im Bereich Berlin durchgeführt. Der Schwerpunkt konzentrierte sich auf die Akzeptanz von Spielen auf mobilen Endgeräten. Es wurde außerdem untersucht, welche Eingabemedien und Nutzerschnittstellen von Nutzern mobiler Endgeräte bevorzugt werden.

Auch wird im Rahmen dieser Arbeit die Entwicklung von Mobiltelefonen und elektronischen Spielen dargestellt.

Hierdurch wird gezeigt welchen Einfluss Spiele auf die Nutzerschnittstellen (MMI) von mobilen Endgerä- ten haben und in Zukunft haben könnten. Es wird außerdem in einem begrenzten Rahmen erörtert, welche Auswirkungen mobile Endgeräte und elektronische Spiele auf die Gesellschaft haben. In diesem Teilbereich soll der Schwerpunkt auf der Nutzung dieser durch Jugendliche liegen.

\section{Technische Entwicklungen}

In diesem Abschnitt wird sowohl auf die Entwicklung elektronischer Spielen als auch auf die mobiler Endgeräte eingegangen. Die Entwicklungsgeschichte wird schwerpunktmäßig erst ab dem Zeitpunkt aufgezeigt, als mobile Endgeräte bzw. elektronische Spiele für ausreichend viele Nutzer verfügbar waren, so dass diese einen spürbaren Einfluss auf die Gesellschaft ausüben konnten. Auf einige gesellschaftliche Veränderungen die dadurch herausgebildet wurden, wird in Kapitel 2 eingegangen. 


\subsection{Mobile Endgeräte}

Es gab natürlich schon mobile Telefonie bevor diese Arbeit mit ihrer Betrachtung beginnt. Einige Beispiele hierfür sind der Zugfunk [1], das Funktelefonsystem A [2], das C-Netz [3], etc. (Eylert 2005: 2ff.) Ende der 80er Anfang der 90er Jahre wurde das D-Netz [4] eingeführt. $\mathrm{Ab}$ diesem Zeitpunkt entwickelte sich das Mobiltelefon stetig weiter zu einem Gerät des täglichen Gebrauchs. Die Digitalisierung der mobilen Endgeräte trug außerdem dazu bei, die Geräte weiter zu miniaturisieren und den Energieverbrauch zu senken.

Mit der Einführung des Nokia 5110 war es erstmals möglich, Spiele auf mobilen Endgeräten zu spielen (dies wird im Kapitel 1.2 Elektronische Spiele genauer beschrieben). Dadurch wurden eine neue Technologie und ein neuer Markt geschaffen, »mobile Games « (elektronische Spiele auf mobilen Endgeräten). Diese Entwicklung beeinflusste sowohl die Gesellschaft als auch die mobilen Endgeräte [5]. Somit hat die Technologie der Mobiltelefone die Technologie der »mobile Games« geformt. Der GSM-Standard wurde daraufhin in Europa eingeführt. Er führte zu einer Vereinheitlichung der mobilen Endgeräte und Netze. Auch nahm die Zahl der Netzteilnehmer stetig zu, so dass ab diesem Zeitpunkt vom Massenmarkt [6] im Bereich des Mobilfunks gesprochen werden kann. (Eylert 2005: 2ff.)

Nach der Einführung von GSM änderten sich die Geräte ständig. Sie wurden immer Nutzerfreundlicher, bekamen Farbdisplays, eine verbesserte Grafikleistung, MP3 Player Funktionen etc. Außerdem entstanden Frameworks und Standards, die das Entwickeln von mobilen Applikationen vereinfachten. All dies verbesserte die Funktionalität und erhöhte die emotionale Bindung zum Nutzer. Auch wurde durch die Verbesserungen im Bereich der mobilen Endgeräte die Verbreitung und Entwicklung von »mobile Games« gefördert.

\section{Beispielhafte Entwicklungsschritte von GSM Telefonen}

Das »International 3200« ist eines der ersten GSM-Mobiltelefone, es ist im Jahr 1992 erschienen. Es besaß ein relativ kleines Display im Vergleich zur Größe, es war hauptsächlich zum Telefonieren gedacht. Das Nokia 5110 war eines der ersten populären Mobiltelefone. Es verfügte als erstes über ein elektronisches Spiel namens »Snake«. Es stellt somit einen Meilenstein in der Entwicklung der »mobile Games« dar. Trotzdem liegt hier der Schwerpunkt noch auf dem Telefonieren. Das Siemens S10 ist das erste Mobiltelefon mit Farbdisplay. Wie noch gezeigt wird, konkurrieren heutige und zu- künftige Mobiltelefone mit mobilen Handheld-Konsolen, wie z. B. dem Gameboy und in Zukunft auch mit Konsolen und Computern im Markt der elektronischen Spiele. Ein entscheidender Faktor um dies zu erreichen waren die Das "Nokia NGage« ist das erste Mobiltelefon das in direkte Konkurrenz mit den bereits erwähnten Handheldkonsolen tritt. Bei diesem Gerät liegt der Fokus auf den elektronischen Spielen. Wie zu erkennen ist, sind die Eingabemedien sehr gut auf Spiele abgestimmt. »Sony Ericsson P800« ist das erste Mobiltelefon, das PDA-Funktionalität mit denen eines Mobiltelefons vereint. Es verfügte bereits über einen Touchscreen. Aus unserer Sicht war dies das erste "Smartphone « und etablierte damit diesen Markt. Für diese Arbeit ist es ein bedeutender Meilenstein, da sich im Markt der Mobiltelefone immer mehr »Smartphones« durchsetzen und auf ihnen unzählige Spiele verfügbar sind.

Zwischen dem nächsten Mobiltelefon (Apple iPhone), das aus unserer Sicht für diese Arbeit bedeutend ist, lagen noch viele weitere Entwicklungen [7]. Deren Hauptaugenmerk lag aber hauptsächlich auf der Kamera-Entwicklung und den Breitbandverbindungen über UMTS. Das »Apple iPhone« erzeugte einen »Hype« [8], dadurch wurden »Smartphones« interessant für den Massenmarkt. Auch andere Anbieter von Hardware zogen auf dieser Produktschiene nach, wie z. B. GoogleAndroid, HTC und andere. Außerdem hat das iPhone Bewegungssensoren und »Multitouchdisplays « für mobile Endgeräte im Massenmarkt etabliert.

Die Innovation auch für »mobile Games« lag nicht nur in dem Gerät selbst sondern auch in der Plattform und dem Umfeld, das für Kunden und Entwickler geschaffen wurde. Auch wurde der Begriff »App« populär gemacht. Er steht im Englischen für eine Software, mit der ein Nutzer eine bestimmte Aufgabe erledigen kann, wie z. B.: eine Taschenrechner Anwendung zum Zusammenrechnen von Zahlen.

Auch wurde ein sogenannter »App-Store« geschaffen, in dem Nutzer Applikationen herunter auf ihr Gerät laden und sofort nutzen können. Außerdem kann jeder Entwickler seine Anwendungen in diesen Hochladen und kostenlos bzw. kostenpflichtig für Nutzer zur Verfügung stellen. Hierbei ist zu beachten dass alle Apps mit jedem »iPhone « und »iPod-Touch « kompatibel sind, da alle das gleiche mobile »Betriebssystem «bzw. die gleiche Plattform nutzen. Durch diese Innovation ist es für den Nutzer einfacher, Applikationen ohne Kompatibilitäts-Probleme zu verwenden. Dies bringt auch Vorteile für den Entwickler; er kann einfacher bzw. billiger An- 
wendungen für alle Geräte dieser Plattform entwickeln und zu vertreiben.

Durch die bereits erläuterte Kombination aus »App«, "App-Store « und einheitlicher Plattform sind viele verschiedene Anwendungen entwickelt worden, unter anderem auch sehr viele mobile Spiele. Auch verbessern sich stetig die Möglichkeiten von mobilen Spielen, da die Hardware ständig weiter entwickelt wird. Wie wir gezeigt haben, sind mobile Geräte bereits heute eine ernst zu nehmende Konkurrenz für Computer, Handheld- und Desktopkonsolen im Spielebereich, da sie ein ständiger Begleiter sind [9]. Ähnliche Möglichkeiten bieten und durch die vielen kostenlosen bzw. kostengünstigen Angebote auch für bisherige Nichtspieler sehr interessant sein können. Die Entwicklung in diesem Bereich ist sicher noch nicht abgeschlossen, weitere zu erwartende und bereits verfügbare Entwicklungen werden im Kapitel 3 erläutert. (Focus 2003; Chip 2007)

\subsection{Elektronische Spiele}

Eines der ersten bekannten elektronischen Spiele wurde im Jahre 1958 von dem amerikanischen Physiker William Higinbotham entwickelt. Es lief auf einem Analogcomputer $\mathrm{ab}$, der mit einem Oszillator verbunden war und als Ausgabegerät diente. Es wurde zu Unterhaltungszwecken innerhalb des Forschungszentrums genutzt.

Eines der ersten populären Videospiele wurde 1972 von einem amerikanischen Studenten Nolan Bushnell entwickelt. Am 29. September 1972 entwickelte er den ersten Videospielautomaten, auf diesem war es möglich eine vereinfachte Variante des Ping-Pongs zu spielen. Dieses Spiel trug den Namen »Pong «. Auf dem Bildschirm des Automaten waren zwei Schläger zu sehen (Weiße Balken auf Schwarzem Hintergrund), sowie ein viereckiger Ball und eine Punkteanzeige. Dieses Spiel erfreute sich großer Beliebtheit. Nolan Bushnell beschloss einen eigenen Automatenvertrieb zu gründen, dieser trug den Namen Atari. Nicht einmal drei Jahre nach dem ersten Pong-Automaten brachte die Firma Atari eine Heimversion (erste Videospielkonsole) von Pong auf den Markt.

In den 80er Jahre im Zuge der Weiterentwicklung der Computertechnik, bekam auch die Videospielindustrie ihren ersten Entwicklungsschub. Viele der heute als Klassiker bezeichneten Spiele wurden zu dieser Zeit entwickelt. Eines der wichtigsten Geräte für das Videospiel war zu dieser Zeit der C64, einer von der Firma Commodore entwickelter Heimcomputer. Dabei etablierte sich das Gerät nicht nur als Arbeitswerkzeug sondern vor allem als Spielkonsole. Aufgrund vieler qualitativ minderwertiger Spiele und der neu entstandenen Mentalität Spiele zu kopieren und nicht zu kaufen, stagnierte der Videospielmarkt allerdings kurze Zeit später. Dies änderte sich erst wieder mit dem Markteintritt des heute weltweit bekannten Videospielkonzerns »Nintendo«. Das Unternehmen, welches anfänglich Spielkarten und anderes Spielzeug produzierte, brachte im Jahr 1983 das »Nintendo Entertainment System«, kurz NES, auf den Markt. Es war eine Spielkonsole mit austauschbaren Spielmodulen. Zum einen war das Kopieren dieser Module nicht mehr für Jedermann ohne größeren Aufwand möglich, zum anderen fanden sich unter den erschienen Spielen einige Titel die sich auch heute noch großer Beliebtheit erfreuen. So zum Beispiel das »Jump-and-Run « »Super Mario Bros.« und das Abenteuerspiel »Zelda«.

Auch Commodore brachte mit dem »Amiga« eine neue Spielekonsole auf den Markt, wie auch Sega, ein japanisches Videospiele-Unternehmen, den »Mega Drive«. Sega entwickelte mit »Sonic« ein weiteres Kultspiel, welches sich großer Beliebtheit erfreute. Damit hatte sich der Videospielmarkt, besonders im Bereich der Videospielkonsolen zu einem wachsenden und von Wettbewerb geprägten Marktsegment entwickelt. In den folgenden Jahren war der Videospielmarkt hauptsächlich von technischen und grafischen Weiterentwicklungen geprägt. Einen entscheidenden Schritt in Richtung mobile Games erlebte die Videospielwelt erst wieder mit der Entwicklung des »Game Boy« (Nintendo 1989).

Mit diesem Gerät wurde die erste mobile Videospielkonsole geschaffen, die es ermöglichte die Spiele in Form von Modulen auszutauschen. Das Gerät war zwar grafisch den inzwischen erhältlichen Heimkonsolen unterlegen, aber es ermöglichte mobile Videospiele. Anfangs war er nur zusammen mit dem Spiel »Tetris« erhältlich, welches sich zu einem der beliebtesten Videospiele aller Zeiten entwickelte. Für den »Game Boy« wurden im Laufe der Zeit über 1200 Spiele entwickelt, unter anderem auch Nintendos Hit-Serien, »Super Mario Land « und »Zelda«, welche nun auch jederzeit und überall spielbar waren.

Mit seinem Marktstart brach das Zeitalter der ubiquitären Unterhaltung an und die Möglichkeit Spiele überall und zu jeder Zeit zu spielen. Aufgrund des großen Erfolges wurde das Gerät im Laufe der Zeit mehrere Male überarbeitet, und in neuer Version mit neuem Funktionsumfang, verbesserter Grafikleistung veröffentlicht. So folgte auf die erste Version des »Game 
Boy« sieben Jahre später der »Game Boy Pocket«, eine kompaktere Version mit verbesserter Anzeige, höherem Kontrast und einer geringeren Energieaufnahme [10]. Zwei Jahre später veröffentlichte Nintendo mit dem »Game Boy Color « die erste Handheld-Konsole welche über eine farbige Anzeige verfügte. Dieses Gerät konnte mehr als 32.000 Farben anzeigen. Aber auch der Heimkonsolenmarkt entwickelte sich weiter, hier finden sich Entwicklungen welche ebenfalls bereits einen Kultstatus erreicht haben. Beispiele hierfür sind die von Sony entwickelte Playstation 1 und 2, welche heute noch die längste Liste an verfügbaren Spielen führen. Dank der Weiterentwicklung der Halbleitertechnik verfügen Konsolen über immer leistungsfähigere Prozessoren und Grafikchips [11].

Die Grafik der heutigen Konsolen wurde dabei in nur wenigen Jahren von schlichten zweidimensionalen Darstellungen, mit 256 Farben, in realistische 3Dimensionale Szenarien weiterentwickelt. Auch HandheldKonsolen werden in Zukunft diese technischen Möglichkeiten intergieren.

\subsection{Entwicklung von Videospielen auf dem Mobiltelefon}

1997 erschien mit dem Nokia 5110 und dem darauf vorinstalliertem Programm »Snake« das erste Videospiel auf einem Mobiltelefon. Der Spieler steuerte eine Schlange (dargestellt als einfache Linie), die auf Punkte zubewegt werden musste, um sie zu verschlingen. Die Schlange wurde nach jedem verschlungenem Punkt ein Stück länger. Die Schwierigkeit bestand darin, dass die Schlange sich nicht selbst berühren durfte, ansonsten endete das Spiel. Obwohl das Spiel inhaltlich wie auch grafisch simpel aufgebaut war, im Gegensatz zu anderen mobilen Spielen, die inzwischen erhältlich waren, wie zum Beispiel auf dem »Nintendo Gamboy«, erregte es ein hohes Aufsehen und wurde international bekannt und beliebt.

Das Spiel wurde daraufhin auch auf jedem weiteren Mobiltelefon der Firma Nokia integriert. Auf Grund der Schlichtheit des Spiels lässt sich die Euphorie darum dadurch erklären, dass es einen erheblichen Mehrwert für das eigene Mobiltelefon darstellte. Es war nun auch möglich, mit diesem mobiles Entertainment zu erleben. Somit entwickelte sich aus der bereits angebrochenen Ära der mobilen Spiele nun das Zeitalter der Handyspiele. Der Kult, der sich um das Spiel "Snake» entwickelte, war eine lange Zeit unübertroffen, da nur wenig vergleichbare Konkurrenz folgte.
Der nächste Entwicklungsschub im Bereich der Handyspiele kam wieder von Nokia. Es kamen mobile Spiele (auf dem Mobiltelefon) auf den Markt, welche durch Farbe und deutlich bessere Grafik geprägt waren. Diese neuen »Handyspiele « waren vergleichbar mit denen auf Konsolen und Handheld-Konsolen. Unter anderem war nun auch das beliebte Spiel »Snake« in Farbe spielbar.

Alle Spiele, die bis dahin auf einem Mobiltelefon spielbar waren, mussten vom Hersteller vorinstalliert werden. Die Möglichkeit andere Spiele hinzuzufügen oder sie auszutauschen bestand nicht. So beschränkte sich das Angebot auf ein paar wenige Spiele, die in Abhängigkeit vom Hersteller variierten. Dies änderte sich erst mit der Einführung der Java-Micro-Edition, kurz Java-ME, im Jahr 1999. Mit diesem Framework wurde es möglich, auf Mobiltelefonen, in der Programmiersprache Java, Spiele und Programme zu entwickeln, zu laden und auszuführen. Dies war ein weiterer entscheidender Schritt in der Entwicklung von »mobile Games«, denn nun war es jedem Entwickler möglich, der diese Programmiersprache beherrschte, Software für ein solches Mobiltelefon zu entwickeln.

Es dauerte nicht lange, bis ein großes Angebot an Videospielen für Java-fähige Mobiltelefone verfügbar war. Diese wurden sowohl von professionellen als auch von Hobbyentwicklern angeboten. Da sich viele Amateurentwickler an der Programmierung und dem Vertrieb von Handyspielen versuchten, war der Markt in kürzester Zeit von einer unüberschaubaren Menge qualitativ minderwertiger Software überflutet. Was nicht zuletzt damit begründet werden kann, dass die Entwickler mit den begrenzten rechentechnischen Ressourcen der Mobiltelefone auskommen mussten. Zudem boten die Zifferntasten, welche zu dieser Zeit das einzige Eingabemedium waren, nur durchschnittlichen Komfort zur Steuerung von Spielen.

Trotzdem wurden Spiele auf Mobiltelefonen immer beliebter, deshalb startete Nokia einen Versuch mit einer weiteren Innovation. Es wurde versucht, auf Mobiltelefonen durch bewusste Anpassung des Designs einen erhöhten Spielkomfort zu gewährleisten. Das aus dieser Überlegung entstandene Gerät war das Nokia »NGage«, das 2003 erstmals auf den Markt kam. Die Bauform ist der einer Handheldkonsole nachempfunden. Es enthält separate Bedienelemente wie z. B. ein Steuerkreuz. Auffällig ist dabei die Ähnlichkeit zum »Gameboy Advanced «, der zu dieser Zeit, aktuellen Handheldkonsole von Nintendo. Die Spiele, die für das »NGage« verfügbar waren, genügten gehobenen Qualitätsstandard im 
Gegensatz zu vorher auf Mobiltelefonen verfügbaren Spielen, so dass diese teilweise mit Spielen für gängige Handheldkonsolen vergleichbar wurden. Das Gerät erzielte allerdings nicht den gewünschten Erfolg, was vermutlich mit dem hohen Preis in Verbindung gebracht werden kann. Weitere Aspekte sind womöglich die geringe Auswahl an Spielen und die Tatsache, dass es nicht einfach möglich war Spiele auszutauschen. Hierfür war es nötig, den Akku zu entfernen. Ein Jahr nach der Veröffentlichung des »NGage« startete Nokia einen weiteren Versuch und stellte das »NGage QD« vor. Dabei wurde beim Design auf die Kritikpunkte des ersten "NGage « eingegangen und so besaß das Gerät z. B. einen separaten Steckplatz, um die Spiele leichter auszutauschen. Allerdings kostete das Gerät noch einmal deutlich mehr als die erste Generation und so blieb auch die neue Version des "NGage« mit seinem Erfolg auf der Strecke. Trotzdem zeichnete sich an dieser Stelle schon deutlich ein neuer Trend ab, der in den folgenden Jahren die Entwicklung neuer Mobilfunkgeräte prägen sollte. Da sich »mobile Games « ungeachtet des Misserfolges von Nokias »Handy-Konsole« einer konstanten Beliebtheit erfreuten, wurde beim Entwurf neuer Mobilfunkgeräte darauf geachtet, inwiefern sich die Geräte als Spielplattform eignen.

Eine Revolution der Videospiele auf dem Mobiltelefon kam im Jahre 2007 mit dem Erscheinen des als iPhone« bezeichneten Smartphones des amerikanischen Computerhersteller Apple. Das Gerät vereinte dabei einige Vorzüge, die es möglich machten, es beinahe als eigenständige Spielkonsole zu bezeichnen, nur ging es bei der Umsetzung einen anderen Weg als die bisherigen Versuche. Die Bedienung des Geräts und dadurch auch die Steuerung von Spielen kann dabei auf unterschiedliche Weise erfolgen. Als Eingabemedium verfügt das Gerät über einen Touchscreen und eine Beschleunigungs- und einen Lagesensor, Tasten entfallen dabei völlig. Eingabemedien wie berührungsempfindliche Anzeigen waren inzwischen auch in der Welt der Handheldkonsolen in Mode gekommen und gestalteten die Landschaft neuer Videospielentwicklungen um. Ein Beispiel hierfür ist das »Nintendo DS «, das als Ablösung für den »Game Boy« zu betrachten ist. Das »iPhone« erweiterte dies nun noch um die benannten Sensoren zur Eingabe. Ein weiterer Punkt der das »iPhone« allerdings gegen bisherige Smartphones abgrenzt, welche schon vorher über Touchscreens verfügten, war, dass es Technologien zur Verfügung stellte, um auch grafisch Anspruchsvolle Spiel dafür entwickeln zu können. Das
Gerät bietet Softwareentwicklern die Möglichkeit mit der offenen Grafik-Programmierschnittstelle »Open GL ES« effizient 3D-Spiele oder grafisch Anspruchsvolle 2D-Spiele zu entwickeln. Diese können, sowie viele andere Anwendungen, dann von Programmierern und Entwicklungsstudios über eine einheitliche Plattform vertrieben werden, den »AppStore«. Diese Plattform bietet dabei Besitzern des »iPhone« nicht nur einen einfachen Weg, neue Spiele zu beziehen, sondern diese auch zu bewerten und zu kommentieren, wodurch sich eine Auswahl qualitativ hochwertigerer Spiele ergibt.

Anders als bei "Nokias NGage« ist auch die Auswahl der Spiele in kurzer Zeit auf ein beachtliches Maß gestiegen, bei rund 30.000 derzeit verfügbaren »iPhone-Apps« ist jedes dritte ein Spiel oder eine unterhaltungsorientierte Anwendung. Dem gleichen Prinzip wie das »iPhone« folgt auch das Handybetriebssystem des Suchmaschinenbetreibers Google, das den Namen »Android « trägt. Anders als das »iPhone» ist »Android « nur eine Softwareplattform für Smartphones. Sie bietet aber ebenfalls eine einheitliche Vertriebsplattform für Apps, den so genannten »Android-Market« und bietet Entwicklern dieselbe Grafik-Programmierschnittstelle an. Die erhältlichen Spiele sprechen dabei verschiedenste Zielgruppen, von Gelegenheitsspielern bis zu Spielern, die auch gern etwas mehr Zeit mit einem Videospiel verbringen, an. Diese sind grafisch so wie auch inhaltlich mit Spielen zeitgemäßer Handheldkonsolen, wie der »Playstation Portable« oder dem Nintendo »DS « zu vergleichen.

\section{Gesellschaftliche Auswirkungen}

Im Folgenden wollen wir auf einige gesellschaftliche Entwicklungen hinweisen, die durch Mobiltelefone, elektronische Spiele und »mobile Games« ausgelöst, bzw. mitgestaltet wurden. Der Einfluss der mobilen Telefone, bzw. der mobilen Kommunikation, machte sich bereits sehr früh in Ideen, Wunschvorstellungen, Literatur und Kunst bemerkbar, auch wenn dieser noch sehr gering war. Ein Beispiel hierfür ist eine literarische Beschreibung durch Erich Kästner in einem Kinderbuch, das bereits 1932 erschien.

Ein Herr, der vor ihnen auf dem Trottoir langfuhr, trat plötzlich aufs Pflaster, zog einen Telefonhörer aus der Manteltasche, sprach eine Nummer hinein und rief: „Gertrud, hör mal, ich komme heute eine Stunde später zum Mittagessen. Ich will vorher noch ins Laboratorium. Wiedersehen, Schatz! «(Kästner 1931) 
Hiermit zeigte Kästner bereits eine eingetretene gesellschaftliche Entwicklung auf, dass nämlich Mobiltelefone hauptsächlich für Kurzgespräche und Absprachen genutzt werden. Es gibt auch noch viele andere Einflüsse von Mobiltelefonen auf die Gesellschaft und das Nutzungsverhalten dieser Geräte. Auf all diese Entwicklungen einzugehen ist im Rahmen dieser Arbeit leider nicht möglich, daher werden wir nur kurz einige Beispiele nennen, und Entwicklungen ansprechen, die in der Zukunft zu erwarten sind.

Einen bedeutenden Einfluss hat das Mobiltelefon z. B. auf Jugendliche und junge Erwachsene. Da sie mit dieser Technologie aufgewachsen sind, hat es maßgeblich ihr Kommunikationsverhalten und ihre Kommunikationskultur beeinflusst. Dieser Aspekt wurde durch Richard Harper und Lynne Hamill bereits ausführlich Untersucht (Hamill et al. 2005, 61ff.). Mobile World. Wie auch von Alex S. Taylor und Jane Vincent dargestellt, hat die Technologie der SMS das Nutzungsverhalten von mobilen Endgeräten massiv beeinflusst. (Taylor et al. 2005: 75ff.)

Das Mobiltelefon ist zu einem täglichem Begleiter und Unterstützer im Arbeits- und Privatleben geworden. Dies wurde z. B. durch die Verbesserung der Geräte [12] und den daraus resultierenden neuen Möglichkeiten erreicht (Lasen 2005; Ling 2004; Wenzel 2007). In der Zukunft ist zu erwarten, dass Mobiltelefone immer mehr zum universellen Freizeitbegleiter weiterentwickelt werden. Dies ist auch schon durch heutige Entwicklungen zu beobachten, da viele Geräte bereits heute mit Mp3-Player, Kamera, »Videoplayer«-Funktionen etc. ausgestattet sind (Heilige 2008). Auch ist in vielen Ländern, z. B. Korea, Japan und Italien, das mobile Fernsehen (mobile TV) bereits akzeptiert und wird von einer hohen Teilnehmerzahl verwendet (Rösch 2009).

Außerdem erwarten wir in der Zukunft, dass mobile Endgeräte zu konkurrenzfähigen mobilen Konsolen, durch eine verbesserte Grafikleistung, mehr angebotene Spiele, etc., weiterentwickelt werden. Es ist anzunehmen, dass diese neue Nutzungsmöglichkeit hauptsächlich von den unter 30-jährigen wahrgenommen wird, da diese Altersgruppe sowohl mit elektronischen Spielen als auch mit mobilen Endgeräten aufgewachsen ist. Auch erwarten wir, dass sich daraus resultierend neue Spielformen entwickeln z. B. »Location Based Multiplayer Games « [siehe auch www.flub.it] [13]. Diese sind als elektronisches Pendent zu Geländespielen, wie der klassischen Schnitzeljagd oder Räuber und Gendarm zu verstehen. Außerdem wird es möglich sein digitale Va- rianten von klassischen Brettspielen wie Scrabble etc. [14] zu spielen. Auch vermuten wir, dass diese mobilen Endgeräte mit ihren vielfältigen elektronischen Spielen die klassischen Konsolen aus dem Markt aus drei Gründen verdrängen:

n Die Hardware ist bei den meisten Jugendlichen bereits vorhanden.

- Die angebotenen elektronischen Spiele sind zum Großteil kostenfrei verfügbar bzw. zu deutlich geringeren Preisen zu erwerben und bieten ähnliche Qualität wie Konsolenspiele.

Deshalb wird diese Arbeit versuchen, in Kapitel 4 und 5 zu untersuchen, ob Jugendliche elektronische Spiele auf mobilen Endgeräten benutzen, ob sie bereit sind, für diese zu bezahlen und ob die Grafikleistung für den Erfolg von diesen Spielen entscheidend sein wird.

\section{Zukünftige Entwicklungen}

Mobiltelefone sind in den vergangenen Jahren immer vielfältiger geworden und haben sich auf Grund der rapiden Entwicklung der Halbleitertechnik zu multifunktionalen Geräten mit beachtlicher Rechenleistung entwickelt. Zusätzlich zur Verschmelzung der Mobiltelefonen mit Kameras, Musikplayern, Fernsehern, Terminplanern und anderen Alltagsgegenständen zeichnet sich deutlich $\mathrm{ab}$, dass der nächste Schritt in der Synthese mit Videospielkonsolen besteht, bzw. schon seit geraumer Zeit im Gange ist.

Geht die technische Entwicklung in den folgenden Jahren mit der gleichen Geschwindigkeit weiter, so könnten uns in Zukunft Mobiltelefone erwarten, welche mit der Rechenleistung jetziger Heimcomputer oder darüber hinaus ausgestattet sind. So soll zum Beispiel der Nachfolger der von Sony entwickelten Handheldkonsole »Playstation Portable« über einen Mehrkernprozessor verfügen, der in einer leistungsfähigeren Variante in der aktuellen »Playstation 3 « verbaut ist, ein so genannter Cell-Prozessor. Es ist abzusehen, dass solche oder ähnliche Prozessoren in nicht allzu ferner Zukunft ihren Weg in Mobilfunkgeräte finden werden. Zudem werden immer neue Eingabemöglichkeiten entwickelt, so wie die aktuell im Gespräch befindliche Gesten-Steuerung. Dadurch soll es möglich sein Endgeräte berührungslos, durch Bewegung der Hände oder mittels der Augen oder ähnlichem, zu bedienen.

Einen weiteren, recht neuartigen Trend beschreiben so genannte »Location Based Games«, welche durchaus 
die Zukunft mobiler Spiele darstellen könnten. Dabei handelt es sich um Spiele, welche auf GPS-gestützten Endgeräten Anwendung finden und die die aus dem GPS gewonnen Positionsdaten auf eine beliebige Art im Spiel integrieren.

\section{Angewendetes Verfahren zur quantitativen Befragung}

Wie bereits in der Einleitung erwähnt, wurde im Rahmen dieser Arbeit eine Umfrage durchgeführt. Im Folgenden soll das Vorgehen erläutert werden, wie diese durchgeführt wurde. Hierbei werden die von uns gestellten Fragen aufgezeigt und erläutert. Die Ergebnisse werden in Kapitel 5 ausgewertet und dem geneigten Leser zur Interpretation überlassen.

Das Ziel war es, mindestens 50 Personen zu Befragen, mit einem möglichst gleichen Anteil von Frauen und Männern. Die Altersgruppe der Befragten sollte zwischen 13 und 65 Jahren liegen, wobei der Schwerpunkt auf Personen zwischen 13 und 30 liegen sollte, da diese Altersgruppe meist bereits Erfahrungen mit elektronischen Spielen und mobilen Endgeräten gesammelt hat. Somit ist zu erwarten, dass sie die Schwerpunktzielgruppe für »mobile Games« sind. Die Befragung wurde in Berlin auf dem Alexanderplatz durchgeführt. Es wurde

\begin{tabular}{|l|l|}
\hline Frage & Fragestellung (Antwortmöglichkeiten) [Anmerkungen] \\
\hline 1 & Nutzen Sie Spiele auf ihrem Mobiltelefon? (ja/nein) \\
\hline 2 & $\begin{array}{l}\text { Wie viele Stunden wöchentlich nutzen Sie durchschnittlich } \\
\text { ihr Mobiltelefon oder andere mobile Endgeräte für ein elekt- } \\
\text { ronisches Spiel? (Stunden) } \\
\text { [auch Social-Games wie z. B. Farmville] }\end{array}$ \\
\hline 3 & $\begin{array}{l}\text { Würden Sie 3d Spiele auf ihrem mobilen Endgerät nutzen? } \\
\text { (ja/nein) }\end{array}$ \\
\hline 4 & $\begin{array}{l}\text { Hätten Sie gerne ein Mobiltelefon mit besserer Grafik- } \\
\text { leistung? (ja/nein) }\end{array}$ \\
\hline 5 & $\begin{array}{l}\text { Welchen Menüstil bevorzugen Sie auf mobilen Endgeräten? } \\
\text { (textbasiert, touch/gesten, kontextstark, kontextschwach) } \\
\text { [Mehrfachnennungen möglich] }\end{array}$ \\
\hline 6 & $\begin{array}{l}\text { Welche Eingabemedien bevorzugen Sie auf mobilen } \\
\text { Endgeräten? } \\
\text { (physische Tastatur, Touchscreen, Bewegungssensor, } \\
\text { separate Bedienelemente ähnlich NGage oder Gameboy) } \\
\text { [Mehrfachnennungen möglich] }\end{array}$ \\
\hline $\begin{array}{l}\text { Sind Sie bereit für Spiele auf mobilen Endgeräten zu } \\
\text { bezahlen? (ja/nein) }\end{array}$ \\
\hline 7
\end{tabular}

sich für diesen Ort entschieden, weil dort ein möglichst breites Spektrum an Personen befragt werden konnte.

Außerdem wurde versucht, die Fragen so zu entwickeln, dass die Befragten nicht durch die Fragestellung beeinflusst wurden.

\subsection{Fragebogen}

In Tabelle 1 sind die Fragen zu sehen, die den Befragten gestellt wurden. Außerdem wurden den Befragten noch Bilder zur Verdeutlichung der Fragen 5 und 6 gezeigt, diese beinhalteten Mobiltelefone mit den entsprechenden Merkmalen aus den Antwortmöglichkeiten.

\begin{tabular}{|c|c|c|c|}
\hline \multicolumn{4}{|c|}{ Befragungsergebnisse } \\
\hline \multirow{2}{*}{\multicolumn{2}{|c|}{ Befragte Personen: 44}} & Frauenanteil: & 23 \\
\hline & & Männeranteil: & 21 \\
\hline \multirow{4}{*}{\multicolumn{2}{|c|}{ Altersverteilung }} & $(13-19)$ & 17 \\
\hline & & $(20-24)$ & 20 \\
\hline & & $(25-35)$ & 5 \\
\hline & & $(35+)$ & 2 \\
\hline & & $\mathrm{Ja}$ & Nein \\
\hline \multirow[t]{3}{*}{ Frage 1} & & $72,7 \%$ & $27,3 \%$ \\
\hline & Frauen & $69 \%$ & $31 \%$ \\
\hline & Männer & $75 \%$ & $25 \%$ \\
\hline \multirow[t]{3}{*}{ Frage 3} & & $52 \%$ & $48 \%$ \\
\hline & Frauen & $43 \%$ & $57 \%$ \\
\hline & Männer & $61 \%$ & $39 \%$ \\
\hline \multirow[t]{3}{*}{ Frage 4} & & $44 \%$ & $56 \%$ \\
\hline & Frauen & $30 \%$ & $70 \%$ \\
\hline & Männer & $56 \%$ & $44 \%$ \\
\hline Frage 7 & & $16 \%$ & $84 \%$ \\
\hline \multirow[t]{2}{*}{ Frage 2} & & Spieldauer im Mittel & 2 Stunden \\
\hline & & Varianz & 9.12744186 \\
\hline \multirow[t]{4}{*}{ Frage 5} & & textbasiert & $20 \%$ \\
\hline & & touch & $72 \%$ \\
\hline & & kontextstark & $47 \%$ \\
\hline & & kontextschwach & $45 \%$ \\
\hline \multirow[t]{4}{*}{ Frage 6} & & physische Tastatur & $20 \%$ \\
\hline & & Touchscreen & $50 \%$ \\
\hline & & Bewegungssensor & $47 \%$ \\
\hline & & $\begin{array}{l}\text { seperate Bedien- } \\
\text { elemente }\end{array}$ & $45 \%$ \\
\hline
\end{tabular}

Tabelle 2 


\section{Auswertung der Befragung}

Im Rahmen dieser Arbeit wurden mit dem zuvor erläuterten Fragebogen 44 Personen befragt, wovon 23 der Befragten weiblich und 21 männlich waren. 95\% der Befragten lagen in der Altersgruppe von 13-35 Jahre. Es gaben $72 \%$ der Befragten an, dass sie elektronische Spiele auf ihrem Mobiltelefon nutzen, wobei die wöchentliche Nutzungsdauer im Mittel mit ca. 2 Stunden angegeben wurde. Es stimmten $52 \%$ der Befragten zu, dass sie 3D-Spiele auf ihrem mobilen Endgeräte spielen würden. 56\% der Teilnehmer der Umfrage gaben an, dass sie keine bessere Grafikleistung auf mobilen Endgeräten wünschen. Hieraus Schlussfolgern wir, dass die meisten Nutzer grafisch weniger aufwendige aber vom Spielprinzip ansprechende Spiele bevorzugen. Nur ca. $16 \%$ der Befragten gaben an das sie bereit wären für elektronische Spiele auf mobilen Endgeräten zu bezahlen.

\section{Fazit}

Wie diese Untersuchung gezeigt hat, benutzen mehr als zwei Drittel der Befragten bereits heute "mobile Games«. Daher kann es sich als Anbieter von solcher Software lohnen, in diesen Markt weiter zu investieren. Da die meisten Befragten nicht bereit sind für »mobile Games« zu bezahlen, sollte aus unserer Sicht darauf geachtet werden, dass dies werbefinanzierte, kostenfreie oder »Shareware«-Angebote, bei denen zusätzliche Inhalte bezahlt werden müssen, sind. Für die meisten Teilnehmer unserer Befragung war die Grafikleistung auf ihrem Mobilgerät nicht entscheidend. Daraus schließen wir, dass die angebotene Hardwareleistung ausreichend und 3d-Grafik in Spielen nicht entscheidend für den Erfolg am Markt ist. Es sollte vor allem darauf geachtet werden, dass die Software per Touchscreen bedient werden kann. Wir konnten nach der Auswertung keinen signifikanten unterschied zwischen Männern und Frauen feststellen. In Zukunft ist aus unserer Sicht zu erwarten, dass Mobiltelefone Handheldkonsolen absorbieren, wie sie es schon mit Digitalkameras, MP3-Player, Navigationssystemen, Organizern, etc. getan haben.

\section{Acknowledgement}

Der Beitrag wurde erstellt im Rahmen des Seminars Telecommunications and Society von Prof. Dr. Bernd Eylert, TH Wildau [FH]

\section{Anmerkungen}

[1] Ab 1918 militärisch genutzt auf der Strecke Zossen - Berlin. Ab 1926 auch Zivil angeboten durch die Deutsche Reichspost und die Deutsche Reichsbahn. Durch die Entdeckung und praktische Umsetzung der Sprachübertragung über Funk ist folgende These aus der Vorlesung bestätigt: These 1: Technologie ist angewandte Naturwissenschaft. (Es musste erst durch Grey und Bell ermöglicht werden Sprache als elektrische Impulse zu Übertragen. Außerdem musste naturwissenschaftlich ein Verfahren entdeckt werden, das es ermöglichte dies über Funk zu tun.)

[2] Ab 1950 in Deutschland eingesetzt.

[3] Ab 1985. Sowohl das A- als auch das C-Netz wurden hauptsächlich von bedeutenden Personen der Wirtschaft, Politik und Gesellschaft genutzt da sowohl die Verbindungskosten als auch die Gerätekosten sehr hoch waren.

[4] Mit dem D-Netz kam der Wandel vom analogen zum digitalen Mobilfunknetz.

[5] Einflüsse hat dies z. B.: auf Design, Benutzung, Nutzerschnittstellen etc.

[6] Das Mobiltelefon wurde ab diesem Zeitpunkt vollen allen Gesell schaftschichten, sowohl von Jungen als auch Alten, genutzt.

[7] Diese sind für diese Studie nicht entscheidend daher wurden sie weggelassen.

[8] Künstlich erzeugtes und überzogenes Interesse für eine Neuentwicklung. Siehe auch »Der Hype-Zyklus stellt dar, welche Phasen der öffentlichen Aufmerksamkeit eine neue Technologie bei deren Einführung durchläuft. Der Begriff des Hype-Zyklus wurde von der Gartner-Beraterin Jackie Fenn geprägt (The Microsoft System Software Hype Cycle Strikes Again, Jackie Fenn, 1995) und dient heute Technologieberatern zur Bewertung in der Einführung neuer Technologien.« (Wikipedia 2010)

[9] "any place, any time, any where (Eylert 2005)

[10] In Japan erschien sogar eine Version des Game Boy Pocket mit hintergrundbeleuchtetem Display

[11] Es befindet sich im Kern der inzwischen neusten Version der Playstation ein so genannter Cell-Prozesser, dieser ist für parallele Rechenarbeiten ausgelegt.

[12] Geräte die sowohl Kalender, Navigationslösungen, Währungsrechner, Taschenrechner, Internetzugriff etc. bieten.

[13] Der englische Begriff Location-based Game (LBG) bezeichnet ein (Computer-)Spiel, in dem der Spielverlauf in irgendeiner Form durch die Veränderung der geografischen Position des Spielers beeinflusst wird. Auf Deutsch bedeutet Location-based Game "positionsbezogenes Spiel«.

[14] Scrabble für iPhone und iPad. http://www.electronic-arts.de/ spiele/scrabble 


\section{Literaturverzeichnis}

Chip (2007): www.chip.de. (Online) (Zitat vom: 20. Mai 2010). http:// www.chip.de/bildergalerie/Meilensteine-Die-besten-Handys-aus15-Jahren-Mobilfunk-Galerie_20204633.html.

Eylert, B. (2005): The Mobile Multimedia Business. Chichester: John Wiley \& Sons Ltd.

Focus (2003): Focus. (Online) (Zitat vom: 20. Mai 2010). http://www. focus.de/digital/handy/handyvergleich/handy-test-ericsson-t68/ ausstattung-das-erste-handy-mit-farbdisplay_aid_308756.html.

Graft, K. (2006): Business Week, http://www.businessweek.com/ innovate/content/jan2006/id20060122_077129.htm (Zugriff 15.5.2010).

Kästner, E. (1931): Der fünfunddreißigste Mai oder Konrad reitet in die Südsee. 55. Hamburg, Dressler Verlag.

Hamill, L., Lasen, A. (2005): Mobile World. United States of America. In: Hamill, L., Lasen, A. (Hrsg) (2005): Mobile World: Past, Present and Future, Springer-Verlag.

Harper, R., Hamill, L. (2005): Kids will be kids: The role of mobiles in teenage life. In: Hamill, L., Lasen, A. (Hrsg) (2005): Mobile World: Past, Present and Future, Springer-Verlag.

Hamill, L., Lasen, A. (Hrsg) (2005): Mobile World: Past, Present and Future, Springer-Verlag.

Taylor, V. (2005): Mobile World: An SMS History. In: Hamill, L., Lasen, A. (Hrsg) (2005): Mobile World: Past, Present and Future, Springer-Verlag.

Ling, R. (2004). The Mobile Connection. San Francisco: Morgan Kaufmann Publishers Elsevier Inc.

Heillige, H. D (Hrsg) (2008): Mensch Computer Interface. Bielefeld: transcript Verlag.

Rösch, A. (2009). magnus.de. (Online). http://archiv.magnus.de/ breitband/artikel/studie-mobiler-tv-empfang-in-italien-waechststark-fussball-wm-als-treiber.html (Zugriff 15.5.2010),

Rosenstingl, H. (2009): Computerspiele und Gesellschaft - Determinanten und Umsetzung des österreichischen Weges der Positivprädikatisierung von Computer- und Konsolenspielen. Master Thesis. s.1.: Donau Universität Krems.

Wenzel, E. (2007): www.zukunftsinstitut.de. http://www.portel. de/index.php?id=nachricht\&no_cache $=1 \&$ tx_ttnews[tt_ news $]=13629 \&$ tx_ttnews[backPid $]=12 \& \mathrm{cHash}=7 \mathrm{f} 9 \mathrm{a} 4 \mathrm{ff} 46 \mathrm{~d}$ (Zugriff 17.5.2010).

Wikipedia (2010): wikipedia.de, http://de.wikipedia.org/wiki/HypeZyklus (Zugriff 26.5.2010).

Authorenangabe:

Johannes Koslowsky B.Eng. Studierender an der TH-Wildau (FH) Studiengang Telematik

Danny Janz B.Eng Studierender an der TH-Wildau (FH) Studiengang Telematik

\section{Autoren}

Johannes Koslowsky, B.Eng.

TH Wildau [FH]

FB Ingenieuerwesen/Wirtschaftsingenieurwesen

Studiengang Telematik

johannes.koslowsky@th-wildau.de

\section{Danny Janz, B.Eng.}

TH Wildau [FH]

FB Ingenieuerwesen/Wirtschaftsingenieurwesen Studiengang Telematik

danny.janz@th-wildau.de 